\title{
An Optimal Control Approach for Deformable Registration
}

\author{
Gabriel L. Hart ${ }^{\dagger}$, Christopher Zach ${ }^{\dagger}$, Marc Niethammer ${ }^{\dagger}, \#$ \\ ${ }^{\dagger}$ Department of Computer Science, \#Biomedical Research Imaging Center \\ University of North Carolina at Chapel Hill \\ $\{$ hartg, cmzach, mn\}@es.unc.edu
}

\begin{abstract}
This paper addresses large-displacement-diffeomorphic mapping registration from an optimal control perspective. This viewpoint leads to two complementary formulations. One approach requires the explicit computation of coordinate maps, whereas the other is formulated strictly in the image domain (thus making it also applicable to manifolds which require multiple coordinate charts). We discuss their intrinsic relation as well as the advantages and disadvantages of the two approaches. Further, we propose a novel formulation for unbiased image registration, which naturally extends to the case of time-series of images. We discuss numerical implementation details and carefully evaluate the properties of the alternative algorithms.
\end{abstract}

\section{Introduction}

Determining point-correspondences between imagepairs based on image information alone is a fundamental problem and of critical importance in image analysis. Image-pairs are assumed to have similarities in structure as well as appearance, where the similarity can for example be the result of looking at temporal phenomena (e.g., at two image frames of a movie) or of investigating relations between two distinct images known a-priori to share largescale structural similarities (e.g., comparing the brains of two subjects). The problem is usually termed image matching or optical flow in computer vision and image registration in medical imaging ${ }^{1}$.

In this work, we propose nonlinear registration schemes within the large-displacement-diffeomorphic mapping (LDDM) setting, derived from optimal control principles. This allows us to obtain an intrinsic (not relying on extrinsic coordinates) registration formulation and to show the connection to previously proposed approaches, against which we carefully compare different numerical implementations. The viewpoint also leads to a novel way to make

\footnotetext{
${ }^{1}$ We use image registration as our terminology of choice in this paper.
}

the estimation problem symmetric and is applicable to manifolds which require multiple coordinate charts.

Registration is based on simultaneously achieving similarity between corresponding image points and imposing spatial regularity on the correspondences. Similarities can be measured by image-intensity differences, mutual information, or cross correlation, to name but a few possibilities. Regularity is achieved by restricting the allowable space of deformations, e.g., by imposing parametric models of image transformation, such as rigid, similarity, or affine transformations, or by non-parametric modeling of image deformation requiring spatially smooth deformation fields. The non-parametric approach is the most flexible, but poses the biggest challenges with respect to the estimation of point correspondences. The estimation problem is infinite-dimensional in the continuum and becomes highdimensional by discretization.

A multitude of regularization approaches has been proposed for non-parametric registration [22, 33, 13]. The two main classes are (i) regularizers operating on the displacement fields (elastic registration) and (ii) regularizers operating on a velocity field (fluid registration). The estimation problems associated with these two classes are

$$
\begin{aligned}
& u=\underset{u}{\operatorname{argmin}} \int \Psi_{s}^{u}[u]+\frac{1}{\sigma^{2}} \Psi_{d}\left[u, I_{0}, I_{1}\right] d \Omega, \quad \text { and } \\
& v=\underset{v}{\operatorname{argmin}} \iint \Psi_{s}^{v}[v] d \Omega d t+\frac{1}{\sigma^{2}} \int \Psi_{d}\left[u, I_{0}, I_{1}\right] d \Omega,
\end{aligned}
$$

where $u$ denotes the displacement field $(\Phi(x)=x+u(x))$, $I_{0}$ and $I_{1}$ are the images to be registered, $v$ is the timedependent velocity field $\left(\partial_{t} \Phi(x, t)=v(x, t)\right), \Omega$ is the spatial domain, $\Psi_{s}^{u}$ and $\Psi_{s}^{v}$ are the regularization measures, and $\Psi_{d}$ is the data attachment term. Choosing

$$
\Psi_{s}^{u}[u]=\sum_{i=1}^{d}\left\|\nabla u_{i}\right\|^{2} \quad \text { and } \quad \Psi_{d}[u]=\left(I_{t}+D I u\right)^{2}
$$

yields Horn and Schunck (HS) optical flow [14], which uses a linearized model for the image match $\left(\psi_{d}[u], D I=\right.$ $\left.(\nabla I)^{T}\right)$ and the Dirichlet energy for the regularization of 
displacements. HS optical flow is one of the simplest elastic registration methods. Since its inception, a variety of improvements have been proposed for optical flow approaches, ranging from nonlinear data attachment terms to piecewise smooth regularization. See $[3,7]$ and references therein. Medical image registration frequently requires bijective maps between images. This may be accomplished explicitly by penalizing folds and singularities during the estimation of the deformation field [12] or implicitly by imposing sufficient regularity on estimated velocity fields [8, 20, 9, 29, 28]. Miller et al. [20, 21] have shown that fluid registration of the form

$$
E(v)=\int_{0}^{1}\|v\|_{V}^{2} d t+\frac{1}{\sigma^{2}}\left\|I_{0} \circ \Phi_{1,0}-I_{1}\right\|_{L_{2}}^{2},
$$

yields diffeomorphic (smooth and invertible) maps $\Phi_{1,0}$ for suitable norms $\|\cdot\|_{V}$ of the velocity field $v$, while allowing for large displacements (by penalizing $v$ instead of $u$ ). Here, $\Phi_{1,0}$ is the velocity-induced image map from $t=0$ to $t=1$.

Estimation problem 2 hinges on the estimation of $\Phi_{0,1}$, which is determined by $v$. In Sec. 2, we give an optimal control interpretation of the estimation problem 2 independent of $\Phi_{0,1}$ and derive its associated optimality conditions. Sec. 3 discusses solution approaches to the optimization problem. Equivalence to the solution method by Beg et al. [4] is established in Sec. 4. Sec. 5 proposes a new approach to render problem 2 symmetric with respect to the registered images. Sec. 6 discusses numerical approaches to solve the image-based optimization problem. Results are given in Sec. 7, including a careful evaluation of the properties of the alternative implementations. Sec. 8 concludes the paper. Mathematical details are given in the Appendix.

\section{Optimal Control Approach}

The optimization problem 2 depends on the velocity field $v$ as well as the velocity-dependent map $\Phi_{0,1}$. This formulation leads directly (upon explicitly deriving the variation of $E(v)$ with respect to $v$ ) to the the map-based optimization approach proposed by Beg et al. [4].

This section shows how we can derive an equivalent result directly in the image domain, without explicitly utilizing the map $\Phi_{0,1}$ using an optimal control approach. An advection equation and a scalar conservation law will be the main parts of the optimality conditions obtained. A global coordinate map $\Phi$ is no longer used, which is a cornerstone for adaptations to manifolds which cannot be covered by a single coordinate chart (such as the sphere). This is for example of particular importance for the analysis of cortical function and structure, where surfaces of spherical topolgy need to be registered. While landmark-based methods exist to compute large-displacement diffeomorphic maps on spherical surfaces, they do not readily extend to more general manifolds or require multiple coordinate charts [11, 2]; and a large-displacement diffeomorphic registration scheme working directly on images has not been investigated so far [31].

Instead of minimizing Eq. 2, we look at image registration from a dynamical systems point of view and solve it as a constrained minimization problem. The system dynamics is the constraint imposed, the velocity field $v$ is regarded as a control input to the dynamical system. The optimization problem is to find the optimal control $v$ subject to the system dynamics with state $I\left(I(0)=I_{0}\right)$, such that image $I(1)$ approximates $I_{1}$. See [19] for background on the optimal control of systems governed by partial differential equations and the rich literature on data assimilation (as used for example in weather forecasting) for details $[23,10]$. The energy to be minimized is then

$$
\begin{aligned}
E(v)= & \int_{0}^{1}\|v\|_{V}^{2} d t+\frac{1}{\sigma^{2}}\left\|I(1)-I_{1}\right\|_{L_{2}}^{2}, \\
\text { s.t. } & I_{t}+(D I) v=0, \quad I(0)=I_{0},
\end{aligned}
$$

which is an advection equation for $I$, governed by the velocity field $v$. Source image, $I_{0}$, and target image, $I_{1}$, are given, the velocity field $v(x, t)$ is sought. Here

$$
\begin{aligned}
\|f\|_{L_{2}}^{2} & =\langle f, f\rangle ;\langle f, g\rangle:=\int f^{*} g d \Omega, \\
\|f\|_{V}^{2} & =\langle f, f\rangle_{V} ;\langle f, g\rangle_{V}:=\langle L f, L g\rangle=\left\langle L^{\dagger} L f, g\right\rangle,
\end{aligned}
$$

where $\Omega$ is the spatial solution domain, $L$ is a differential operator (e.g., $L=-\alpha \nabla^{2}+\gamma$ ) and $L^{\dagger}$ denotes its adjoint. Choosing an appropriate differential operator $L$ ensures a diffeomorphic transformation [9]. Minimizing energy 3 subject to constraint 4 is equivalent to minimizing

$$
\begin{aligned}
E(v, I, \lambda, \gamma) & =\int_{0}^{1}\|v\|_{V}^{2}+\left\langle\lambda, I_{t}+(D I) v\right\rangle d t \\
& +\left\langle\gamma, I(0)-I_{0}\right\rangle+\frac{1}{\sigma^{2}}\left\|I(1)-I_{1}\right\|_{L_{2}}^{2}
\end{aligned}
$$

with respect to $v(x, t), I(x, t)$, and the two Lagrangian multipliers $\lambda(x, t), \gamma(x)$ [27]. For a candidate minimizer of the unconstrained energy, its variations with respect to $v, \lambda, I$, and $\gamma$ need to vanish, which yields the optimality conditions (see Appendix A for a derivation)

$$
\begin{aligned}
I_{t}+(D I) v & =0 \\
-\lambda_{t}-\operatorname{div}(\lambda v) & =0 \\
2 L^{\dagger} L v+(D I)^{T} \lambda & =0 \\
I(0) & =I_{0}, \\
\lambda(1) & =\frac{2}{\sigma^{2}}\left(I_{1}-I(1)\right) .
\end{aligned}
$$

The Lagrangian multiplier $\gamma$ disappears due to the fixed initial condition for $I$. Eq. 6 is the prescribed state equation 
(the system dynamics), Eq. 7 is the adjoint or co-state equation, Eq. 8 is the compatibility condition, and Eqs. 9 and 10 are the initial condition for the image and the final condition for the adjoint variable $\lambda$ respectively. The final condition $\lambda(1)$ for the adjoint variable measures the error between the given image $I_{1}$ and image $I_{0}$ in the warped coordinate system of time $t=1$. The adjoint equation 7 is a scalar conservation law, i.e., it flows the image error of Eq. 10 backward in time while conserving the overall error measure. Since

$$
\delta E(v ; d v)=\int_{0}^{1}\left\langle 2 L^{\dagger} L v+(D I)^{T} \lambda, d v\right\rangle d t,
$$

the compatibility condition 8 equals the gradient of the energy with respect to the velocity in the $L_{2}$ norm

$$
\nabla_{v}^{L_{2}} E(v)=2 L^{\dagger} L v+(D I)^{T} \lambda .
$$

As in the map-based solution approach by Beg et al. [4], or for the evolution of Sobolev active contours [26], improvements in convergence by gradient descent in Sobolev space may be obtained for adjoint solution methods [25]. The gradient with respect to the norm $V$ induced by $L$ is

$$
\nabla_{v}^{V} E(v)=2 v+\left(L^{\dagger} L\right)^{-1}\left((D I)^{T} \lambda\right) .
$$

The overall algorithm proceeds by: (i) flowing image $I_{0}$ forward in time, (ii) computing the current error at time $t=$ 1, (iii) flowing the error backward in time, subject to the scalar conservation law for the adjoint variable, and by (iv) performing a gradient descent step, where the gradient for any time $t \in[0,1]$ can be computed from the state and the adjoint variables. See Fig. 1 for the overall algorithm.

Borzi et al. [6] propose a similar approach for optical flow computations. They use the same system dynamics, but a fundamentally different regularization of the velocity field $v$. Their solution method thus requires the solution of an elliptic partial differential equation (across space and time) in addition to the solution of the state and the adjoint equations. In contrast, the approach in this paper does not explicitly regularize over time, which makes it easier to compute and allows (due to equivalence of the solution to the solution obtained by the approach of Beg et al. [4], see Sec. 4) for diffeomorphic deformations if desired. Of note, the work by Papadakis and Memin [24] uses variational data assimilation for the tracking of curves and fluid motions. However, while their approach is based on a weak constraint formulation (using the control function to allow for deviations from the nominal dynamics), we enforce the dynamical model strictly, and control the velocity $v$.

\section{Solutions for the State and its Adjoint}

Two equations play a central role in the optimal control formulation for registration: The advection equation

$$
I_{t}+D I v=0, \quad I(0)=I_{0}
$$

and the scalar conservation law for the adjoint variable

$$
-\lambda_{t}-\operatorname{div}(\lambda v)=0, \quad \lambda(1)=\lambda_{1} .
$$

The former flows image intensities along the velocity field $v$, the latter flows the matching error along $v$, while conserving its overall mass. Given a current estimate of the velocity field $v$, the current estimate for the map $\Phi_{t, 0}$ is governed by

$$
\Phi_{t}+D \Phi v=0, \quad \Phi(0)=i d,
$$

an advection equation for $\Phi$. The solution to Eq. 12 is then

$$
I(t)=I_{0} \circ \Phi(t) .
$$

Computing the inverse map $\Phi^{-1}=\Phi_{t, 1}$ as

$$
-\Phi_{t}^{-1}-D \Phi^{-1} v=0, \quad \Phi^{-1}(1)=i d
$$

we have for any infinitesimal volume element $d V$

$$
\left|D \Phi^{-1}(t)\right| d V(t)=d V(1) .
$$

Regarding $\lambda$ as a mass density, conservation of mass implies

$$
\lambda(1) \circ \Phi^{-1}(t) d V(1)=\lambda(t) d V(t)
$$

which is

$$
\lambda(1) \circ \Phi^{-1}(t) d V(1)=\lambda(t) \frac{1}{\left|D \Phi^{-1}(t)\right|} d V(1),
$$

and allows expressing $\lambda(t)$ with respect to $\lambda(1)$ as

$$
\lambda(t)=\left|D \Phi^{-1}(t)\right| \lambda(1) \circ \Phi^{-1}(t) .
$$

Sec. 4 shows how these relations connect the image-based approach of Sec. 2 to the map-based approach by Beg et al. [4]. Sec. 6 discusses the numerical ramifications of choosing between the formally equivalent image-based and map-based approaches. Of note, the same equivalence relation can be applied to the approach by Borzi et al. [6].

\section{Relation to Previous Approach}

The map-based solutions of the advection equation and the scalar conservation law given in Sec. 3 allow for the design of a hybrid solution approach. Here, the forward and the inverse maps are solved for numerically. Once obtained, they provide an analytic solution to the state variable $I$ and its adjoint $\lambda$, which allows for the computation of the energy gradient with respect to the time-dependent velocity field $v$ :

$$
\begin{aligned}
\nabla_{v}^{V} E(v)= & 2 v+\left(L^{\dagger} L\right)^{-1}(D I(t))^{T} \lambda(t) \\
= & 2 v+\frac{2}{\sigma^{2}}\left(L^{\dagger} L\right)^{-1}\left(\left|D \Phi^{-1}(t)\right|\right. \\
& \times(D I(t))^{T}\left(I_{1} \circ \Phi^{-1}(t)-I(t)\right) \\
= & 2 v+\frac{2}{\sigma^{2}}\left(L^{\dagger} L\right)^{-1}\left(\left|D \Phi^{-1}(t)\right|\right. \\
& \times\left(D\left(I_{0} \circ \Phi(t)\right)\right)^{T}\left(I_{1} \circ \Phi^{-1}(t)-I(t)\right)
\end{aligned}
$$




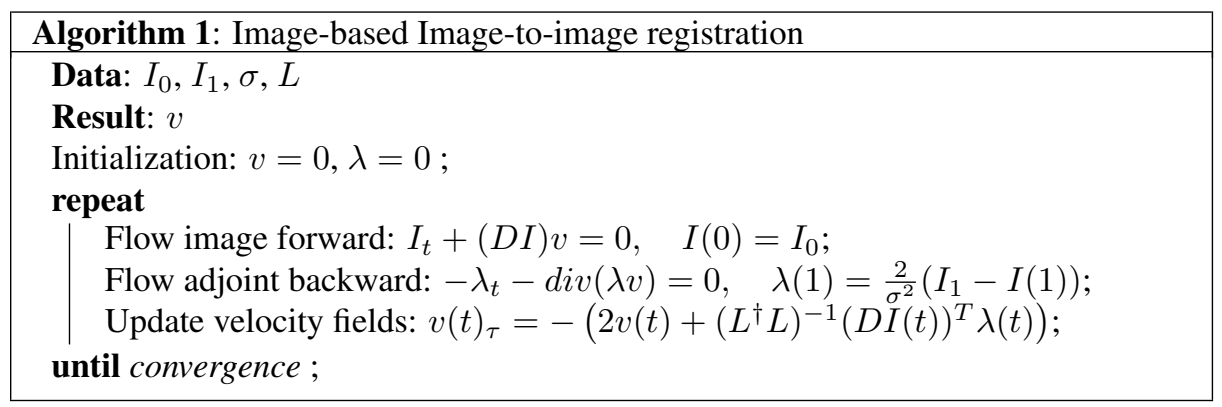

Figure 1. Image-based image-to-image registration and advection results (right). Right: Translating a checkerboard square diagonally using different numerical methods. The 1st order accurate method blurs the image extensively. Methods with higher order numerical accuracy produce much crisper images. WENO5 additionally suppresses undesirable oscillations.
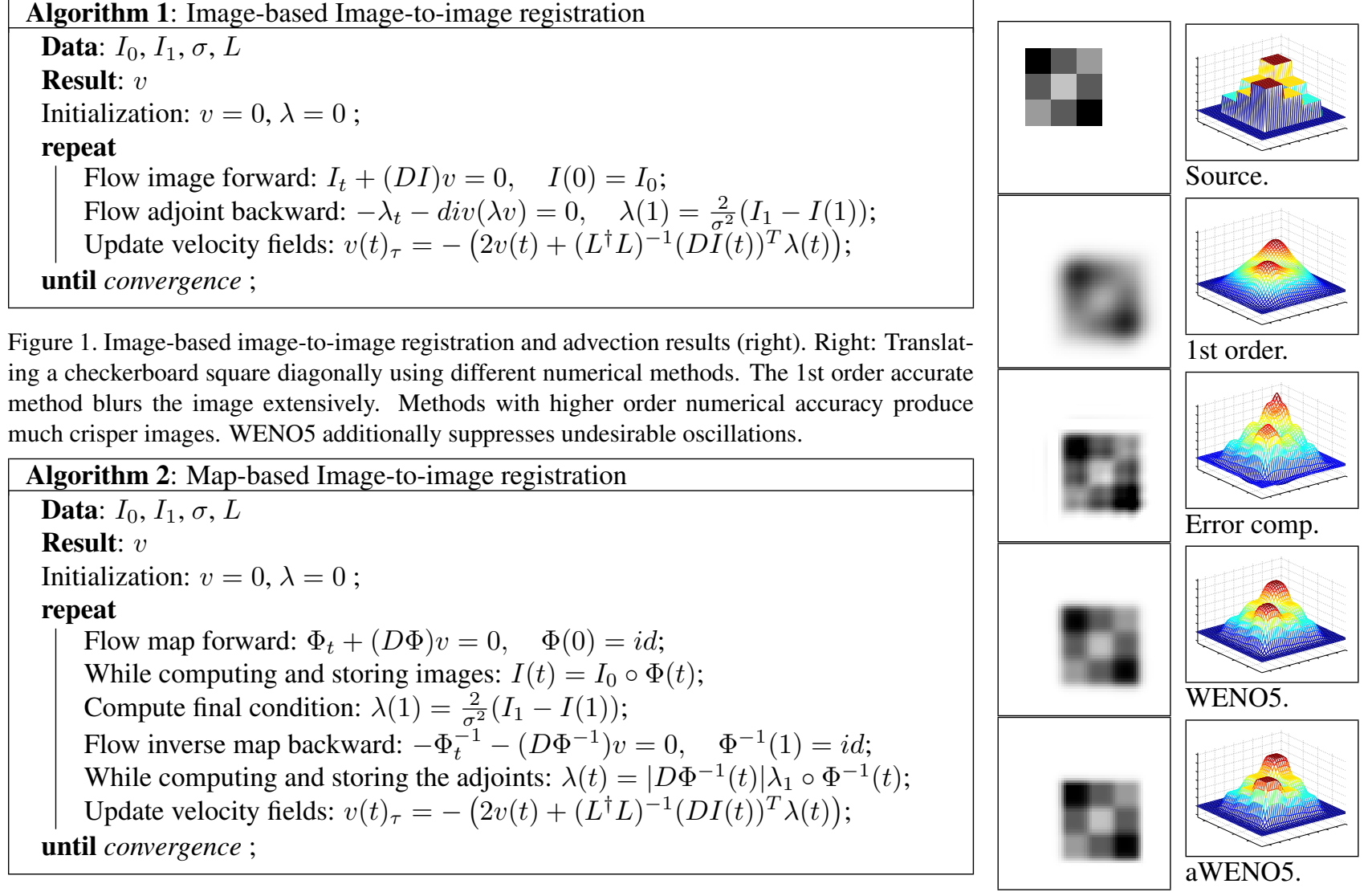

Figure 2. Map-based image-to-image registration.

where Eq. 15 is the original gradient as obtained from the image-based optimal control derivation, Eq. 16 is the gradient as obtained by Beg et al. [4] directly from the mapbased energy of Eq. 2, and Eq. 17 is the fully map-based formulation used for the map-based implementation. This establishes the formal equivalence between the image-based and the map-based approaches. Of note, the image-based scheme neither requires the explicit computation of the forward and the backward maps $\Phi$ and $\Phi^{-1}$ nor image interpolation based on these coordinate transforms. While removing the explicit dependence on a map $\Phi$ leads to a more compact formulation, accurate solutions require care with respect to the numerical method used. See Sec. 6 for details. The overall algorithm is given in Fig. 2.

\section{Unbiased Formulation}

Image registration approaches are frequently not symmetric with respect to the images to be registered. Thus, given two images $\mathrm{A}$ and $\mathrm{B}$, registering $\mathrm{A}$ to $\mathrm{B}$ is not assured to give the inverse result to registering $\mathrm{B}$ to $\mathrm{A}$. To remove this registration bias, optimization problems need to be symmetric. One solution is to simply replace the registration energy by a sum of the original energy and its com- plement. In the context of large displacement diffeomorphic flows, approaches have been proposed by Avants [1], Younes [32] and Beg [5]. The image mismatch can be computed at the midpoint in time $[5,32,1]$

$$
\left\|I_{0} \circ \Phi_{\frac{1}{2}, 0}-I_{1} \circ \Phi_{\frac{1}{2}, 1}\right\|_{L_{2}}^{2}
$$

which amounts to the estimation of two separate flow fields. Alternatively, reweighting the image difference [32]

$$
\int\left|D \Phi_{1,0}^{-1}\right|\left(I_{0} \circ \Phi_{1,0}-I_{1}\right)^{2} d x
$$

or defining a matching cost over time [5]

$$
\int_{0}^{1}\left\|I_{0} \circ \Phi_{t, 0}-I_{1} \circ \Phi_{t, 1}\right\|_{L_{2}}^{2} d t
$$

symmetrizes the registration problem and thus makes it unbiased. Only the consistent integral cost of Eq. 18 readily extends to the case of time-series (where no natural midpoint exists). However, the consistent integral cost leads to 
a gradient of the form [5]

$$
\begin{aligned}
& \nabla_{v}^{V} E=2 v+\frac{2}{\sigma^{2}}\left(L^{\dagger} L\right)\left(\left(I_{1} \circ \Phi_{t, 1}-I_{0} \circ \Phi_{t, 0}\right)\right. \\
& \times\left[D\left(I_{0} \circ \Phi_{t, 0}\right)^{T} \int_{t}^{1}\left|D \Phi_{t, u}\right| d u\right. \\
&\left.\left.\quad+D\left(I_{1} \circ \Phi_{t, 1}\right)^{T} \int_{0}^{t}\left|D \Phi_{t, u}\right| d u\right]\right)
\end{aligned}
$$

which is costly to compute due to the time integrals. In our approach, we directly symmetrize the data attachment term, but combine this with an estimation of the optimal matching template. While the original formulation was an optimal control problem with free final state subject to a final state penalty, the symmetrized version has a free initial as well as a free final state subject to initial and final state penalties. The approach has the following properties: (i) it directly generalizes to time-series of images, (ii) the estimation is performed in one continuous interval, and (iii) the formulation extends to data defined on a manifold that cannot be covered with one coordinate chart. The optimal control problem is then to minimize

$E(v)=\int_{0}^{1}\|v\|_{V}^{2} d t+\frac{1}{\sigma^{2}}\left\|I(0)-I_{0}\right\|_{L_{2}}^{2}+\frac{1}{\sigma^{2}}\left\|I(1)-I_{1}\right\|_{L_{2}}^{2}$,

subject to

$$
I_{t}+D I v=0, \quad I(0)=I_{T},
$$

with an unknown (free) initial state $I(0)=I_{T}$. Due to the formal equivalence of the image-based and the map-based approaches this is identical to minimizing ${ }^{2}$

$E\left(v, I_{T}\right)=\int_{0}^{1}\|v\|_{V}^{2} d t+\frac{1}{\sigma^{2}}\left\|I_{T}-I_{0}\right\|_{L_{2}}^{2}+\frac{1}{\sigma^{2}}\left\|I_{T} \circ \Phi_{1,0}-I_{1}\right\|_{L_{2}}^{2}$

The variation with respect to $I_{T}$ is

$\delta E\left(I_{T} ; d I_{T}\right)=\frac{2}{\sigma^{2}}\left\langle I_{T}-I_{0}+\left|D \Phi^{-1}\right|\left(I_{T}-I_{1} \circ \Phi^{-1}\right), d I_{T}\right\rangle$

which yields

$$
I_{T}=\frac{I_{0}+\left|D \Phi^{-1}(0)\right| I_{1} \circ \Phi^{-1}(0)}{1+\left|D \Phi^{-1}(0)\right|} .
$$

The update equation constitutes a weighted average between the initial image $I_{0}$ and the warped image $I_{1}$. The weighting assures that differences are measured in their respective coordinate systems (for $t=0$ and $t=1$ ). The overall estimation scheme alternates between registration (using $I_{T}$ as the initial condition for the state equation) as before and an update of the template image $I_{T}$ according to

\footnotetext{
${ }^{2}$ The same result can be obtained by strictly working in the image domain. However, the derivation is more complicated, due to the interdependency of image perturbations at different time points.
}

Eq. 19. To make the update independent of the map $\Phi^{-1}$, we can make use of relation 14 , to get

$$
\begin{aligned}
\left|D \Phi^{-1}(t)\right|=q(t), & -q_{t}-\operatorname{div}(q v)=0, q(1)=1, \\
& -J_{t}-D J v=0, \quad J(1)=I_{1} .
\end{aligned}
$$

The updated template image is then

$$
I_{T}=\frac{I_{0}+q(0) J(0)}{1+q(0)},
$$

allowing for an image-based, unbiased registration.

\section{Numerical Considerations}

The image-based approach is potentially more desirable for intensity-based image-matching, since (i) it extends to manifolds without explicit construction of a coordinate system, (ii) it may require less computations (subject to the numerics used), and (iii) it can serve as a proof of concept for image-based algorithms with more complicated dynamics, where the map-based relations of Sec. 4 no longer hold.

Avoiding the computations of the maps $\Phi$ and $\Phi^{-1}$ removes the need for explicit image interpolations. Both the forward and the inverse map are smooth by construction. However, the image $I$ and the adjoint $\lambda$ will generally show significantly more local variation (creating edge information, which is the basis of registration itself). Preserving this local variation on coarse grids is challenging.

To accurately capture the evolutions of the image and the adjoint, requires numerical methods which show minimal numerical diffusion (and thus minimal image blurring). This can be achieved by nonlinear numerical dis.cretizations, e.g., using flux limiters [18], essentially nonoscillatory schemes [16], or schemes aiming at a diffusion reduction [30,17], but may not always be necessary as shown in Sec. 7. Fig. 1 demonstrates the effect of different numerical schemes implementing the advection equation to translate a synthetic checkerboard image. A first order upwinding discretization blurs the image greatly. Higher order accurate numerical schemes (back and forth error compensation, the 5th order weighted essentially non-oscillatory (WENO5) scheme and its anti-diffusive (aWENO5) version) result in significantly less image blurring. By design, the WENO schemes suppress image oscillations. The visually best result is achieved by aWENO5.

Fig. 5 shows computation results for the determinant of the Jacobian of an exemplary map $\Phi$ and the respective adjoint versions according to Eq. 20. As is the case for the advections (Fig. 1) the first order accurate numerical solution provides qualitatively correct, but blurry results. The WENO5 result obtained by solving the scalar conservation law is visually indistinguishable from the direct computation based on the deformation map. 
As a compromise between simplicity, computational complexity, and accuracy, we use WENO5 for our registration experiments: the Hamilton Jacobi scheme for the advection equation [15] and a conservative scheme for the adjoint equation [16]. Time integration is achieved by 3rd order total variation diminishing Runge Kutta. To compare, we also compute the registrations using first order upwinding with Euler forward. We use a first order Roe upwinding scheme with entropy fix for the adjoint equation [18].

\section{Results}

Since the image-based and the map-based approaches are theoretically equivalent (Eqs. 2 and 3), we compare results by computing the resulting energies after convergence of the optimization. To fairly compare the approaches, we assess the quality of the resulting estimated velocity field, $v$, by computing the final registered image for each method by a single WENO5 map pass. We compare image- and map-based approaches using the first order upwinding Euler forward and the WENO5 implementations.

We conducted experiments on a set of synthetic datasets as well as for real brain images. For the synthetic tests, we created two scenarios: one corresponding to a translating square (E cases) (see Fig. 3) and one where the source image had two additional small squares that were not present in the target image and could thus not be matched exactly (I cases). For each scenario, we created three sub-cases to test the implementations' sensitivity to blurred edges and noise. We ultimately ran six synthetic tests: E1 and I1 had crisp edges, E2 and I2 were blurred, and E3 and I3 were blurred and altered with random additive noise.

The synthetic results show that, as expected, both mapbased cases perform slightly better when crisp edges are present. When blurring and additive noise are induced, the disparities between the image-based and map-based results diminish. When considering an impossible registration, the final energy values are higher. The differences between these energy values for the four methods, however, stay relatively constant. Fig. 7 shows an overview of the results.

We also tested our implementations on three pairs of corresponding brain slices taken from two distinct brain volumes (Fig. 4). For this real data, the displacement fields are significantly more regular, and as such the disparity between the results for the different methods diminishes.

To illustrate the unbiased registration approach we created a synthetic test image with nonuniform appearance (Fig. 6). The unbiased registration correctly aligned the image, while estimating a meaningful template image, reminiscent of the mean of the source and the target. The template image was initialized with the source image. Fig. 6 shows the first and the second template updates. No significant change is noticeable after the second iteration.

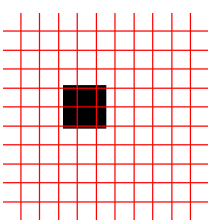

Source.

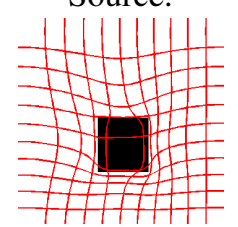

Target.

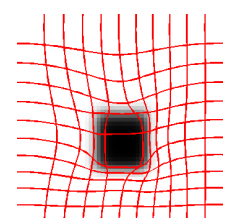

1 st order image.

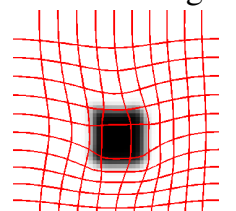

WENO5 image.

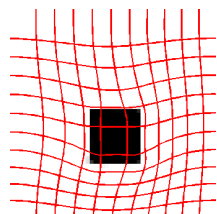

1st order map.

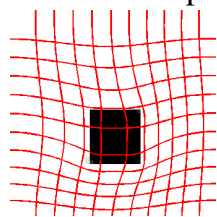

WENO5 map.
Figure 3. Synthetic registration results for test case E1 (see Fig. 7). For large synthetic distortions, the differences between the computed results become more apparent than for the real test images.

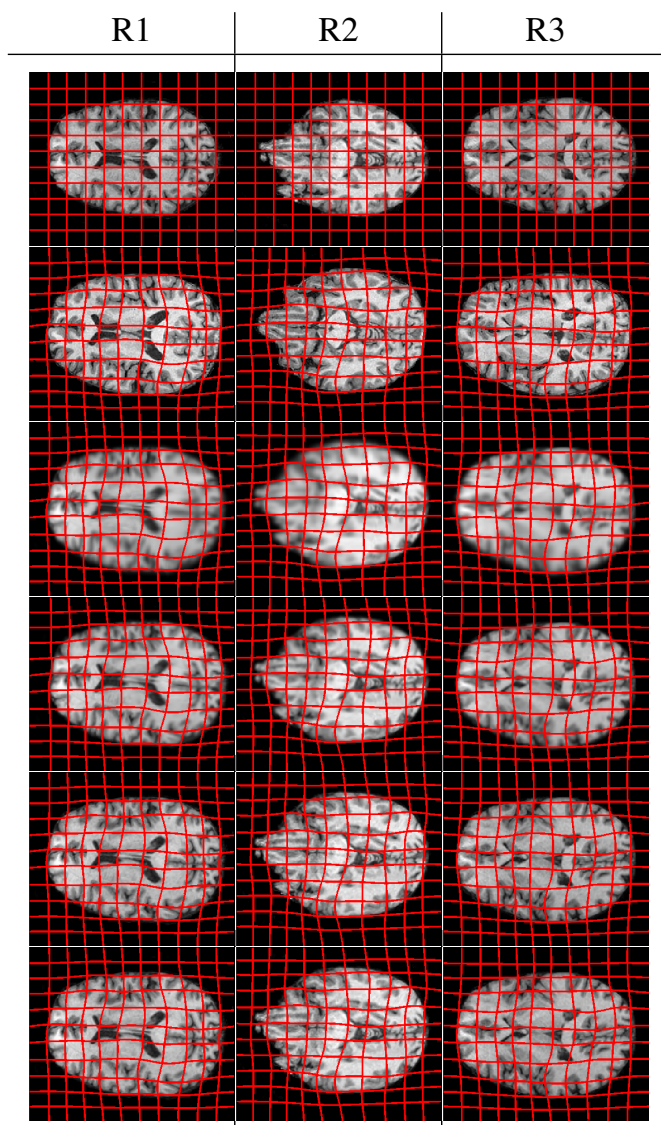

Figure 4. Registration results for real test cases R1, R2, and R3 (see Fig. 7). In descending order for each column: source, target, 1st-order image, WENO5 image, 1st-order map, WENO5 map.

\section{Conclusion and Future Work}

We proposed an optimal control formulation for deformable image registration. We showed the formal equiv- 


\begin{tabular}{c||c|c|c||c|c|c||c|c|c} 
& E1: 8.74 & E2: 5.30 & E3: 5.92 & I1: 10.11 & I2: 5.68 & I3: 6.34 & R1: 4.47e-1 & R2: 3.91e-1 & R3: 4.57e-1 \\
\hline 1st Img & $1.31 \mathrm{e}-1$ & $1.64 \mathrm{e}-1$ & $6.27 \mathrm{e}-1$ & 1.49 & $5.65 \mathrm{e}-1$ & 1.03 & $3.39 \mathrm{e}-1$ & $2.73 \mathrm{e}-1$ & $3.26 \mathrm{e}-1$ \\
5th Img & $4.11 \mathrm{e}-1$ & $3.15 \mathrm{e}-2$ & $4.89 \mathrm{e}-1$ & 1.78 & $4.16 \mathrm{e}-1$ & $8.84 \mathrm{e}-1$ & $3.87 \mathrm{e}-1$ & $2.72 \mathrm{e}-1$ & $3.25 \mathrm{e}-1$ \\
1st Map & $8.36 \mathrm{e}-2$ & $3.60 \mathrm{e}-2$ & $4.95 \mathrm{e}-1$ & 1.46 & $4.20 \mathrm{e}-1$ & $8.96 \mathrm{e}-1$ & $3.39 \mathrm{e}-1$ & $2.74 \mathrm{e}-1$ & $3.27 \mathrm{e}-1$ \\
5th Map & $3.59 \mathrm{e}-2$ & $3.05 \mathrm{e}-2$ & $4.83 \mathrm{e}-1$ & 1.41 & $4.15 \mathrm{e}-1$ & $9.44 \mathrm{e}-1$ & $3.39 \mathrm{e}-1$ & $2.73 \mathrm{e}-1$ & $3.27 \mathrm{e}-1$
\end{tabular}

Figure 7. Final energy results for synthetic test cases E1, E2, E3, I1, I2, I3 (resolution 50 x 50), and real brain images R1, R2, and R3 (resolution $208 \times$ 175). The E cases can be exactly matched, while the I cases are impossible to match perfectly. Cases E1 and I1 have crisp edges, E2 and I2 are blurred, and E3 and I3 are blurred and modified with random additive noise. The initial energy for each test case is given with the label. In the impossible cases, the final energies all increase relative to the exact cases, but the discrepancies between the map-based and image-based registrations remain similar, showing that the relative error discrepancy gets smaller. The three $\mathrm{R}$ cases are corresponding slices from two different brain volumes and hence cannot be matched perfectly.
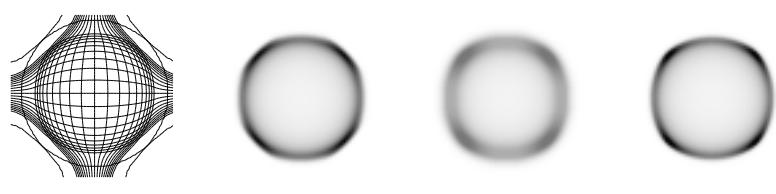

Figure 5. Determinant of Jacobian of $\Phi$. Left to right: Warped grid, $\Phi,|D \Phi|$ map, $|D \Phi|$ 1st-order, and $|D \Phi|$ WENO5. WENO5 produces a faithful result by solving a scalar conservation law. The 1 st-order approach matches qualitatively, but introduces blurring.

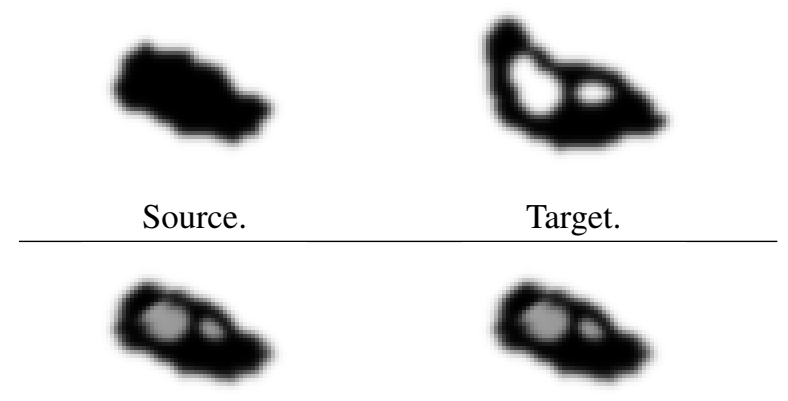

Template 1st iteration. Template second iteration.

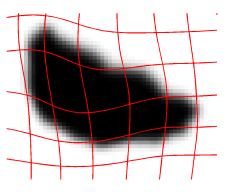

Warped Source.

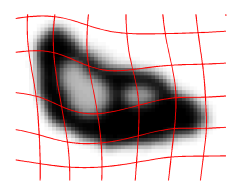

Warped Template.
Figure 6. The unbiased registration approach aligns the images, while estimating a template image reminiscent of an image-mean.

alence between this approach and the registration method proposed by Beg et al. [4], We demonstrated the practicality of purely image-based registrations and conducted a careful analysis of numerical implementations, in comparison to the standard map-based implementations. In comparison to the map-based approach, the proposed image-based approach can be readily extended to manifolds, which so far had not been explored in the large-displacement diffeomorphic image registration framework. We further proposed a new method for unbiased image registration, which generalizes to image sequences. The work suggests the feasibility of similar image-based optimal control approaches to dynamical systems of higher order, which we will explore in future work. Such higher-order dynamical systems are needed to formulate registration as an initial value problem, thereby reducing memory requirements.

\section{A. Optimality Conditions}

For a minimizer of energy 5 , its variations with respect to $v, \lambda, I$, and $\gamma$ need to vanish. Computing

$$
\begin{aligned}
& \delta E(v, I, \lambda, \gamma ; d v, d I, d \lambda, d \gamma)= \\
& \left.\quad \frac{\partial}{\partial \epsilon} E(v+\epsilon d v, I+\epsilon d I, \lambda+\epsilon d \lambda, \gamma+\epsilon d \gamma)\right|_{\epsilon=0},
\end{aligned}
$$

yields

$$
\begin{aligned}
\delta E=\int_{0}^{1} 2\langle v, d v\rangle_{V}+\langle d \lambda, & \left.I_{t}+(D I) v\right\rangle \\
+\left\langle\lambda, d I_{t}+(D d I) v+(D I) d v\right\rangle & d t+\left\langle d \gamma, I(0)-I_{0}\right\rangle+\langle\gamma, d I(0)\rangle \\
+ & \frac{2}{\sigma^{2}}\left\langle I(1)-I_{1}, d I(1)\right\rangle .
\end{aligned}
$$

Since

$$
\int_{0}^{1}\left\langle\lambda, d I_{t}\right\rangle d t=\int_{0}^{1}\left\langle-\lambda_{t}, d I\right\rangle d t+\left.\langle\lambda, d I\rangle\right|_{0} ^{1}
$$

and (by Green's theorem)

$$
\langle\lambda,(D d I) v\rangle=\langle-\operatorname{div}(\lambda v), d I\rangle+\int_{\partial \Omega} d I \lambda v \cdot d S=\langle-\operatorname{div}(\lambda v), d I\rangle
$$

assuming $v$ vanishes on the domain boundary, we get

$$
\begin{gathered}
\delta E=\int_{0}^{1}\left\langle 2 L^{\dagger} L v+(D I)^{T} \lambda, d v\right\rangle+\left\langle I_{t}+(D I) v, d \lambda\right\rangle \\
+\left\langle-\left(\lambda_{t}+\operatorname{div}(\lambda v)\right), d I\right\rangle d t+\left.\langle\lambda, d I\rangle\right|_{0} ^{1} \\
+\frac{2}{\sigma^{2}}\left\langle I(1)-I_{1}, d I(1)\right\rangle+\left\langle I(0)-I_{0}, d \gamma\right\rangle+\langle\gamma, d I(0)\rangle .
\end{gathered}
$$


Collecting terms yields

$$
\begin{gathered}
\delta E=\int_{0}^{1}\left\langle 2 L^{\dagger} L v+(D I)^{T} \lambda, d v\right\rangle+\left\langle I_{t}+(D I) v, d \lambda\right\rangle \\
+\left\langle-\left(\lambda_{t}+\operatorname{div}(\lambda v)\right), d I\right\rangle d t+\left\langle\frac{2}{\sigma^{2}}\left(I(1)-I_{1}\right)+\lambda(1), d I(1)\right\rangle \\
+\left\langle I(0)-I_{0}, d \gamma\right\rangle+\langle\gamma-\lambda(0), d I(0)\rangle .
\end{gathered}
$$

Since $\delta E$ needs to vanish for any $d v, d I, d \gamma, d \lambda$, fulfilling the boundary conditions (i.e., $d I(0)=0$ ) of the problem, the necessary optimality conditions follow.

\section{References}

[1] B. B. Avants. Symmetric diffeomorphic image registration with cross-correlation: Evaluating automated labeling of elderly and neurodegenerative brain. MEDIA, 12:26-41, 2008. 4

[2] M. Bakircioglu, S. Joshi, and M. Miller. Landmark matching on brain surfaces via large deformation diffeomorphisms on the sphere. In Proceedings of the SPIE, pages 710-715, 1999. 2

[3] S. S. Beauchemin and J. L. Barron. The computation of optical flow. ACM Computing Surveys, 27(3):433-467, 1995. 2

[4] M. Beg, M. Miller, A. Trouvé, and L. Younes. Computing Large Deformation Metric Mappings via Geodesic Flows of Diffeomorphisms. IJCV, 61(2):139-157, 2005. 2, 3, 4, 7

[5] M. F. Beg and A. Khan. Symmetric data attachment terms for large deformation image registration. IEEE TMI, 26(9):1179-1189, 2007. 4, 5

[6] A. Borzi, K. Ito, and K. Kunisch. Optimal control formulation for determining optical flow. SIAM Journal on Scientific Computing, 24(3):818-847, 2002. 3

[7] A. Bruhn, J. Weickert, and C. Schnörr. Lucas/Kanade meets Horn/Schunck: Combining local and global optical flow methods. IJCV, 61(3):211-231, 2005. 2

[8] G. E. Christensen, R. D. Rabbitt, and M. I. Miller. Deformable templates using large deformation kinematics. IEEE TIP, 5(10):1435-1447, 1996. 2

[9] P. Dupuis, U. Grenander, and M. Miller. Variational problems on flows of diffeomorphisms for image matching. Quarterly of Applied Mathematics, 56(3):587-600, 1998. 2

[10] G. Evensen. Data Assimilation: The Ensemble Kalman Filter. Springer, 2007. 2

[11] J. Glaunès, M. Vaillant, and M. Miller. Landmark matching via large deformation diffeomorphisms on the sphere. Journal of Mathematical Imaging and Vision, 20(1):179-200, 2004. 2

[12] E. Haber and J. Modersitzki. Image registration with guaranteed displacement regularity. IJCV, 71(3):361-372, 2007. 2

[13] M. Holden. A review of geometric transformations for nonrigid body registration. IEEE TMI, 27(1):111-128, 2008. 1

[14] B. K. P. Horn and B. G. Schunck. Determining optical flow. Artificial Intelligence, 17:185-203, 1981. 1
[15] G.-S. Jiang and D. Peng. Weighted ENO schemes for Hamilton-Jacobi equations. SIAM Journal on Scientific Computing, 21(6):2126-2143, 2000. 6

[16] G.-S. Jiang and C.-W. Shu. Efficient implementation of weighted ENO schemes. Journal of Computational Physics, 126:202-228, 1996. 5, 6

[17] B. Kim, Y. Liu, I. Llamas, and J. Rossignac. Advections with significantly reduced dissipation and diffusion. IEEE TVCG, 13:135-144, 2007. 5

[18] R. J. LeVeque. Finite Volume Methods for Hyperbolic Problems. Cambridge, 2002. 5, 6

[19] J. L. Lions. Optimal Control Systems Governed by Partial Differential Equations. Springer, 1970. 2

[20] M. Miller. Computational anatomy: shape, growth, and atrophy comparison via diffeomorphisms. Neuroimage, 23:S19S33, 2004. 2

[21] M. Miller, A. Trouvé, and L. Younes. On the metrics and Euler-Lagrange equations of computational anatomy. Annual Reviews in Biomedical Engineering, 4(1):375-405, 2002. 2

[22] J. Modersitzki. Numerical Methods for Image Registration. Oxford, 2004. 1

[23] I. M. Navon. Data Assimilation for Atmospheric, Oceanic, and Hydrologic Applications, chapter Data Assimilation for Numerical Weather Prediction: A Review. Springer, 2008. 2

[24] N. Papadakis and E. Mémin. A variational technique for time consistent tracking of curves and motion. Journal of Mathematical Imaging and Vision, 31(1):81-103, 2008. 3

[25] B. Protas, T. R. Bewley, and G. Hagen. A computational framework for the regularization of adjoint analysis in multiscale PDE systems. Journal of Computational Physics, 195:49-89, 2004. 3

[26] G. Sundaramoorthi, A. Yezzi, and A. C. Mennucci. Coarseto-fine segmentation and tracking using Sobolev active contours. IEEE TPAMI, 30(5):851-864, 2008. 3

[27] J. L. Troutman. Variational Calculus and Optimal Control. Springer, 1996. 2

[28] G. Unal and G. Slabaugh. Estimation of vector fields in unconstrained and inequality constrained variational problems for segmentation and registration. Journal of Mathematical Imaging and Vision, 31(1):57-72, 2008. 2

[29] T. Vercauteren, X. Pennec, A. Perchant, and N. Ayache. Non-parametric diffeomorphic image registration with the demons algorithm. In Proceedings of MICCAI, volume 4792, pages 319-326. Springer, 2007. 2

[30] Z. Xu and C.-W. Shu. Anti-diffusive high order WENO schemes for Hamilton-Jacobi equations. Methods and Applications of Analysis, 12(2):169-190, 2005. 5

[31] B. Yeo, M. Sabuncu, T. Vercauteren, N. Ayache, B. Fischl, and P. Golland. Spherical demons: Fast diffeomorphic landmark-free surface registration. Technical report, MIT, 2008. 2

[32] L. Younes. Jacobi fields in groups of diffeomorphisms and applications. Quarterly of Applied Mathematics, 65:113134, 2007. 4

[33] B. Zitova and J. Flusser. Image registration methods: a survey. Image and Vision Computing, 21:977-1000, 2003. 1 